\title{
ANALYSIS OF MODULAR DCM FLYBACK CONVERTERS IN INPUT PARALLEL CONNECTIONS WITH PARAMETRIC MISMATCHES
}

\author{
Vinícius B. Fuerback ${ }^{1}$, Matheus S. Dall'Asta ${ }^{1}$, Mauro André Pagliosa ${ }^{2}$, Telles B. Lazzarin ${ }^{1}$ \\ ${ }^{1}$ Power Electronics Institute (INEP-UFSC), Federal University of Santa Catarina, Florianópolis, SC - Brazil \\ ${ }^{2}$ Federal Institute of Education, Science and Technology Catarinense (UFSC), Luzerna, SC - Brazil \\ e-mail: vinicius.fuerback@gmail.com, dallastamatheus@gmail.com,mauro.pagliosa@luzerna.ifc.edu.br, telles@inep.ufsc.br
}

\begin{abstract}
This paper analyzes the effects of parameter mismatches in the balance mechanism of modular DC-DC Flyback converters operating in discontinuous conduction mode. The natural current and voltage distributions among modules are evaluated when mismatches on duty cycles, transformer magnetizing inductances and transform turns ratios are present. From these results, the critical values of inductances and duty cycles that assure the discontinuous operation are equated. The smallsignal equivalent circuit for Input-Parallel-Output-Series and Input-Parallel-Output-Parallel connections are found, followed by a simple control strategy. The theoretical analysis is verified by experimental results obtained with a prototype composed of three $200 \mathrm{~W}$ Flyback modules, with a rated power of $600 \mathrm{~W}$ and maximum efficiency of $95.5 \%$. Results corroborate the proposed equations for the steady state balance and dynamic behavior of both connections, highlighting the modular characteristic of the converter.
\end{abstract}

\section{Keywords - Flyback, IPOP, IPOS, Modular.}

\section{INTRODUCTION}

Modular converters have been researched for solving problems linked to current or voltage stresses over semiconductors, as well as for improving reliability in several applications [1]-[3]. In DC-DC converters, isolated modular solutions are gaining interest for low-, medium- and high-voltage applications, driven by factors such as increased demand for energy from renewable sources [4], [5], solid state transformers (SST) [6], the expansion of micro-grid distribution systems [7], [8] and the necessity to meet medium and long-distance direct current projects [9].

One of the current challenges in DC-DC converters is to increase the rated power and current/voltage levels without putting down efficiency and reliability. Semiconductors with higher blocking voltage have higher switching losses, especially when operated at high frequency [10], and new semiconductors development is presented as an expensive solution. The power semiconductors arrangements that reduce electrical stresses require the use of additional circuits, which should ensure the equalization of electrical stresses among switches during the blocking step [11], making these options unattractive. Facing these limitations, a viable solution is the development of new arrangements/topologies capable of meeting current and voltage stresses using conventional

Manuscript received 04/12/2018; first revision 03/02/2019; accepted for publication 23/05/2019, by recommendation of Editor Marcello Mezaroba. http://dx.doi.org/10.18618/REP.2019.2.0052 semiconductors [6]. In this context, DC-DC converter modularization is an alternative, once different associations lead to reduced electrical stresses over modules [12].

The Flyback converter is widely used for low-power applications due to its robustness, simplicity and easy control schema. At the same time, the discontinuous conduction mode (DCM) operation greatly simplifies the control strategy [13]. However, the operation in DCM has the disadvantage of high current peak values on both sides of the transformer when processing the same load as in continuous conduction mode (CCM) [14]. It implies in switches and transformer sized for higher peak and effective current values. Then, the Flyback converter operating in DCM tends to be limited to a power of few hundreds of Watts.

Modular connections allow sharing current and/or voltage efforts, making the DCM operation feasible for higher power levels. Another advantage is the possibility of achieving high voltage gain even when a transformer with close to unity turns ratio is used. It usually leads to lower leakage inductances, improving the converter efficiency and reducing the overvoltage over the switches. However, the application of a modular solution requires care regarding the power distribution among modules. Sharing of currents for parallel arrangement and voltages in series connection shall be ensured. Parametric variations among the modules, such as delays in the command signals of the switches, turns ratio of the isolating transformers, the series inductance values and the tolerance values in active and passive components can unbalance of the processed power, causing instability on the operation of the modular system [15], [16].

The balance among modules can be obtained through a decentralized strategy. It implies in controlling electrical quantities in the modules, which improves the steady-state and dynamics behavior of the converter [17], [18]. However, it normally drives to more complex control strategies, increasing the number of sensors and affecting reliability. The natural balance mechanism, or self-balance as is named in the literature, is an ability to find stable operation point, even if the modules are processing different amounts of power [19]. These converters can operate with a common command signal, avoiding multiple control meshes.

There are four different connections: the Input-ParallelOutput-Series (IPOS), Input-Parallel-Output-Parallel (IPOP), Input-Serie-Output-Series (ISOS) and Input-Serie-OutputParallel (ISOP). Each of them has its features and is more suitable for distinct applications. In [1] it is shown that the ISOS DCM Flyback converter has the ability to balance electrical stresses over modules. A similar analysis is conducted for the ISOP in [20]. The ISOS CCM Flyback 
converter is also explored in [21]. However, the literature presents a gap for input parallel DCM Flyback connections.

The IPOP connection is applied at low voltage and high current at both input and output of the converter, once it provides the sharing of the current among modules [22]. Data processing centers and telecommunications are typical applications for this type of connection. On the other hand, the IPOS connection is indicated when voltage boosting is required. Some applications include photovoltaic systems, fuel cells, electrical vehicle systems and UPS [23]-[25].

The current work shows that the current and voltage balance ability of the modular Flyback converter is also inherent in the IPOS and IPOP connections, which are shown in Figures 1.a and 1.b, respectively. Also, it shows how parametric variations among modules affect the sharing of electrical efforts. The following sections prove the stated ideas analytically and experimentally.

In section II, a steady state analysis of both connections is approached, leading to equations that describe the sharing of electrical quantities among modules when parametric variations exist. Section III examines the limits of magnetizing inductance and duty cycle that assure the operation in DCM. A control-oriented modelling is made in Section IV, from which the models for controlling the converter output voltage in both associations are derived. Section V presents simulation and experimental verification. Finally, conclusions are listed.

\section{MODULAR CONNECTION OF FLYBACK CONVERTERS IN DCM}

The voltage gain $G$ represents the ratio between the average output voltage $V_{o}$ and the average input voltage $V_{i}$. In DCM Flyback converter, $G$ depends on the duty cycle $d$, the switching frequency $f_{s}$, the magnetizing inductance $L_{m}$ and on the load $R_{o}$.

$$
G=\frac{V_{o}}{V_{i}}=d \sqrt{\frac{R_{o}}{2 f_{s} L_{m}}} .
$$

Consequently, these parameters influence the sharing of electrical quantities for modular connections. This section aims to quantify the impact of each of them in the distribution of processed power over modules.

\section{A. Steady State Analysis for the Input Parallel Connections}

This analysis considers $N$ DCM Flyback modules in a parallel input connection. The average value of the input current $i_{S_{k}}$ in the module $k$, named $I_{S_{k}}$, is determined by (2).

$$
I_{S_{k}}=\frac{V_{i_{k}}}{2 L_{m_{k}} f_{s}} d_{k}^{2}
$$

In parallel connections, the voltages $V_{i_{k}}$ over the modules are equal to $V_{i}$. Then, isolating the input voltage in (2) for all the modules leads to (3).

$$
I_{S_{1}} f_{s} \frac{L_{m_{1}}}{d_{1}^{2}}=\ldots=I_{S_{k}} f_{s} \frac{L_{m_{k}}}{d_{k}^{2}}=\ldots=I_{S_{N}} f_{s} \frac{L_{m_{N}}}{d_{N}^{2}} .
$$

Taking (3) and making the ratio between $I_{S_{k}}$ and the average input current $I_{S_{j}}$ of the module $j$, one obtains (4), that is

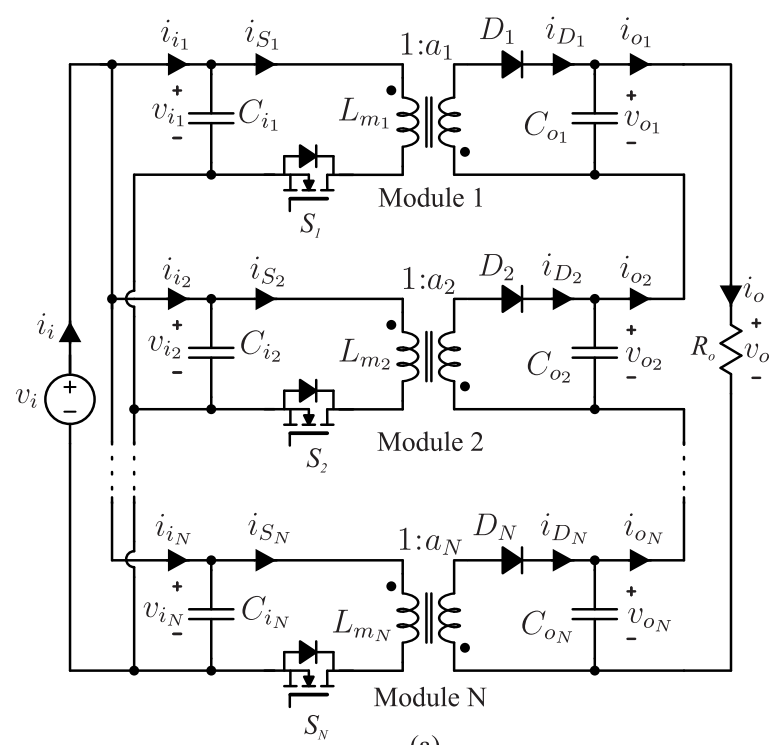

(a)

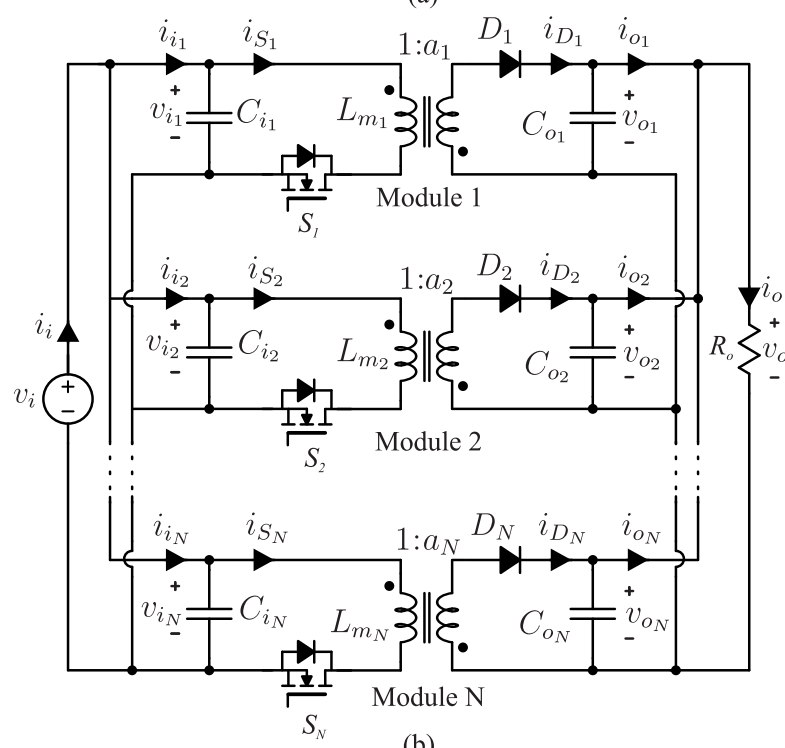

(b)

Fig. 1. IPOS (a) and IPOP (b) modular Flyback connections.

expressed for $I_{S_{j}}$

$$
I_{S_{j}}=I_{S_{k}} \frac{L_{m_{k}}}{L_{m_{j}}}\left(\frac{d_{j}}{d_{k}}\right)^{2} .
$$

In parallel connections, the whole converter input current $i_{i}$ is equal to the sum of the input currents $i_{i_{j}}$ of each module. Assuming the converter operates in steady state, the average value of $i_{i_{j}}, I_{i_{j}}$, is equal to $I_{S_{j}}$. Thus,

$$
I_{i}=\sum_{j=1}^{N} I_{i_{j}}=\sum_{j=1}^{N} I_{S_{j}} .
$$

Applying (4) in (5) and noting that $I_{S_{j}}=I_{i_{j}}$, follows that:

$$
I_{i}=\sum_{j=1}^{N} I_{i_{k}} \frac{L_{m_{k}}}{L_{m_{j}}}\left(\frac{d_{j}}{d_{k}}\right)^{2}=I_{i_{k}}\left(1+\frac{L_{m_{k}}}{d_{k}^{2}} \sum_{\substack{j=1 \\ j \neq k}}^{N} \frac{d_{j}^{2}}{L_{m_{j}}}\right) .
$$

Reorganizing (6), it is obtained the relation among the 
average values of the input currents of a module $k$ and the converter, which describes the steady state current sharing.

$$
\frac{I_{i_{k}}}{I_{i}}=\frac{1}{1+\frac{L_{m_{k}}}{d_{k}^{2}} \sum_{\substack{j=1 \\ j \neq k}}^{N} \frac{d_{j}^{2}}{L_{m_{j}}}} .
$$

\section{B. Steady State Analysis for Output Connections}

This analysis considers $N$ Flyback modules with their outputs in a series connection. In steady state the average values of the currents on the secondary sides of the transformers for all modules are equal. It allows to write (8), which relates the conduction time on diodes $k$ and $j$, given by $t_{2_{k}}$ and $t_{2 j}$, with their peak current values $\hat{i}_{D_{k}}$ and $\hat{i}_{D_{j}}$.

$$
\frac{t_{2 j}}{t_{2_{k}}}=\frac{\hat{i}_{D_{k}}}{\hat{i}_{D_{j}}} .
$$

The output voltage of a module is written as (9), where $a_{k}$ is the transformer turns ratio of the module $k$.

$$
V_{o_{k}}=\frac{\hat{i}_{D_{k}}}{t_{2_{k}}} L_{m_{k}} a_{k}^{2}
$$

The output voltage of the modules $k$ and $j$ are related by:

$$
\frac{V_{o_{k}}}{V_{o_{j}}}=\frac{L_{m_{k}}}{L_{m_{j}}} \frac{t_{2_{j}}}{t_{2_{k}}} \frac{\hat{i}_{D_{k}}}{\hat{i}_{D_{j}}}\left(\frac{a_{k}}{a_{j}}\right)^{2} .
$$

Applying (8) in (10) and noting that the peak current in the primary side of the transformer for the module $k$ is given by $\hat{i}_{S_{k}}=\hat{i}_{D_{k}} a_{k},(11)$ is written.

$$
\frac{V_{o_{j}}}{V_{o_{k}}}=\frac{L_{m_{j}}}{L_{m_{k}}}\left(\frac{\hat{i}_{S_{j}}}{\hat{i}_{S_{k}}}\right)^{2} .
$$

The relation $\hat{i}_{S_{j}} / \hat{i}_{S_{k}}$ depends on the input connection. For an input parallel converter, it is given by:

$$
\frac{\hat{i}_{S_{j}}}{\hat{i}_{S_{k}}}=\frac{d_{j}}{d_{k}} \frac{L_{m_{k}}}{L_{m_{j}}} .
$$

Substituting (12) in (11) yields:

$$
\frac{V_{o_{j}}}{V_{o_{k}}}=\frac{L_{m_{k}}}{L_{m_{j}}}\left(\frac{d_{j}}{d_{k}}\right)^{2} .
$$

The output voltage $V_{o}$ is the sum of each module output voltage $V_{o_{j}}$, resulting in:

$$
V_{o}=\sum_{j=1}^{N} V_{o_{j}}=\sum_{j=1}^{N} V_{o_{k}} \frac{L_{m_{k}}}{L_{m_{j}}}\left(\frac{d_{j}}{d_{k}}\right)^{2} .
$$

By equating (14), (15) is found, which relates the average output voltage values between the converter and one module.

$$
\frac{V_{o_{k}}}{V_{o}}=\frac{1}{1+\frac{L_{m_{k}}}{d_{k}^{2}} \sum_{\substack{j=1 \\ j \neq k}}^{N} \frac{d_{j}^{2}}{L_{m_{j}}}} .
$$

A similar analysis can be applied for the output parallel connection, considering the relation among the output currents on the modules. For the IPOP association, the imbalance is given by:

$$
\frac{I_{o_{k}}}{I_{o}}=\frac{1}{1+\frac{L_{m_{k}}}{d_{k}^{2}} \sum_{\substack{j=1 \\ j \neq k}}^{N} \frac{d_{j}^{2}}{L_{m_{j}}}} .
$$

For both IPOS and IPOP associations the turns ratios do not affect the balance. A simple way to comprehend it is referring to the average value of the output current of a single module. Assuming the DCM operation, $a$ affects solely the peak value and conduction time of $i_{D_{k}}$. Its average value $\left(I_{D_{k}}\right)$ remains the same regardless of the turns ratio of the converter. The balance equation relates average values over a switching period, making it independent of $a$. The conclusions are similar to the averaged output voltage of a single module.

\section{Unbalance Sensibility}

It is observed that (7), (15) and (16) are similar. Then, they are referred by a general equation named as $u_{k}$. It represents the fraction of voltage or current (for series or parallel connections, respectively) that each module processes in a modular association. If the parameters of all modules are the same, then it is obtained the ideal value of $u_{k}$ :

$$
u_{k, \text { ideal }}=\frac{1}{N}
$$

The effect of parameter mismatches over electric efforts is verified by calculating how much power is processed by a module in comparison to an ideal case, where all of them are equal. It is represented as:

$$
\Delta u_{k}=\frac{u_{k}-u_{k, \text { ideal }}}{u_{k, \text { ideal }}}=\left(u_{k}-\frac{1}{N}\right) N .
$$

Varying the magnetizing inductance from $-10 \%$ to $+10 \%$ in relation to the other modules and verifying how much the current or voltage processed diverges from the average value, the curve shown in Figure 2.a is obtained. An equivalent analysis is done for the duty cycle, as shown in Figure 2.b.

It should be noticed from Figure 2 that connections with higher number of modules have higher percentage imbalances. For input parallel connections, the module that has the lowest value of magnetizing inductance has the highest current stress in the primary side. This results in higher voltage levels for this module in an output series connection and a higher output current value in the case of output parallel connection. For the duty cycle analysis, it is shown that the module with higher conduction time processes more energy, which means it has the highest currents and voltage values for parallel and series output connections, respectively.

A relevant situation would be the use of decentralized 


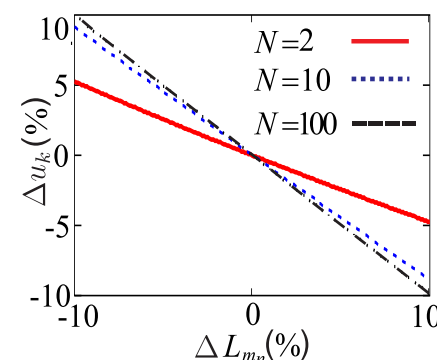

(a)

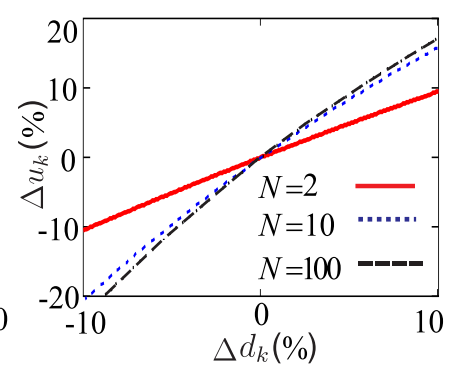

(b)
Fig. 2. Unbalance sensibility for a percent variation of $\pm 10 \%$ a) in the magnetizing inductance; b) in the duty cycle.

control, where the duty cycles are imposed in such a way that deviations on voltage or current among modules are fully compensated. From Figure 2, an increment $L_{m_{j}}$ could be balanced by increasing the duty cycle. However, it would require the precise knowledge of all inductance mismatches or the measurement of currents in output parallel connections or voltages in output series connections for each module that compose the converter. Normally, the deviations are small when compared to the efforts for reducing them to zero.

\section{CRITICAL VALUES FOR DCM OPERATION}

The sharing of electrical efforts and the control strategy relies on the DCM operation. However, there are limits for parametric mismatch to assure this conduction mode. The maximum magnetizing inductance value for the DCM operation for one Flyback module is given by:

$$
L_{m_{k}}=\frac{V_{o_{k}}}{I_{o_{k}}} \frac{\left(1-d_{k}\right)^{2}}{2 a_{k}^{2} f_{s}} .
$$

For an IPOP connection, the voltage $V_{o}$ is common to all modules, while the output current is given by (16). Thus,

$$
L_{m_{k}}=\frac{V_{o}}{I_{o}} \frac{\left(1-d_{k}\right)^{2}}{2 a_{k}^{2} f_{s}}\left(1+\sum_{\substack{j=1 \\ j \neq k}}^{N} \frac{L_{m_{k}}}{L_{m_{j}}}\left(\frac{d_{j}}{d_{k}}\right)^{2}\right) .
$$

Equating (20) for $L_{m_{k}}$ yields (21), that presents the maximum magnetizing inductance value in which the DCM is assured.

$$
L_{m_{k}}=\frac{R_{o}}{\frac{2 a_{k}^{2} f_{s}}{\left(1-d_{k}\right)^{2}}-\frac{R_{o}}{d_{k}^{2}} \sum_{\substack{j=1 \\ j \neq k}}^{N} \frac{d_{j}^{2}}{L_{m_{j}}}} .
$$

The turns ratio $a_{k}$ does not affect directly the balance, however it limits the magnetizing inductance. Increasing $D_{k}$ reduces $L_{m_{k}}$ as well, imposing limits for a decentralized control. The increase of $L_{m_{j}}$ turns the DCM more stable, once the range of $L_{m_{k}}$ becomes larger. Finally, a decrease on the output power $\left(P_{o}\right)$ increases the limit of $L_{m_{k}}$. Naturally, the added module should be projected to process at least $P_{o} / N$.

For output parallel connections, the maximum value of duty cycle that ensures the operation in DCM for one specific module $k$ regardless of its inductance value is:

$$
d_{k}=\frac{1}{1+\sqrt{\frac{2 a^{2} f_{s}}{R_{o} \sum_{\substack{j=1 \\ j \neq k}}^{N} \frac{d_{j}^{2}}{L_{m_{j}}}}}} .
$$

The relation shown in (22) is depicted in Figure 3 for $N$ modules with same magnetizing inductance $L_{m}$. It should be noted that when a higher number of modules are connected in parallel, the system becomes more robust in terms of maintaining the operation in DCM, since the critical duty cycle is increased with more modules.

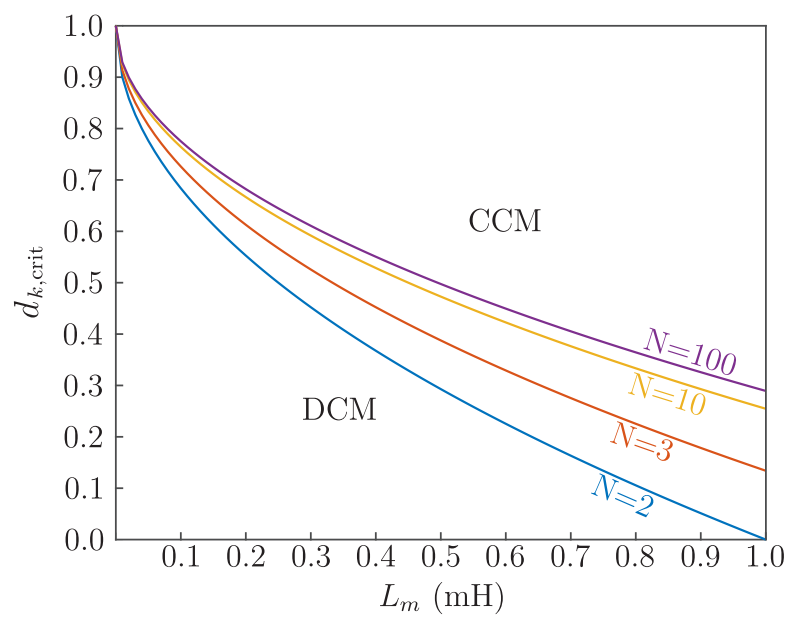

Fig. 3. Critical duty cycle value of one module to guarantee its operation in DCM based on the number of modules $N$ and the magnetizing inductances $L_{m}$.

When the duty cycle is higher than the critical value $D_{k}$, (21) results on a positive and valid value. As depicted in Figure 4 for three modules with duty cycle $D_{k}=D_{1}=D_{2}=D_{3}$ and magnetizing inductances $L_{m_{1}}=320 \mu \mathrm{H}, L_{m_{2}}=400 \mu \mathrm{H}$ and $L_{m_{3}}=480 \mu \mathrm{H}$, the value of the critical inductance $L_{m_{k \text {.crit }}}$ decreases when the duty cycle increases. Also, the critical inductances are different among the three modules.

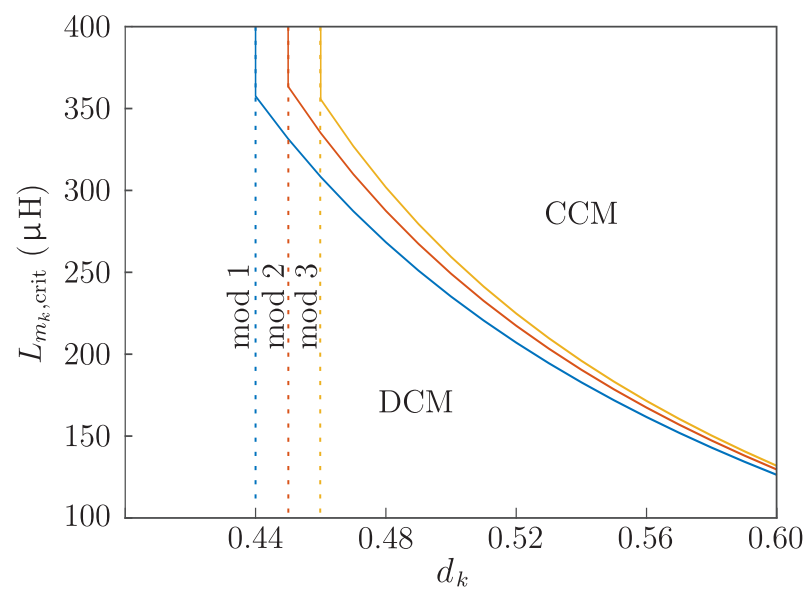

Fig. 4. Critical magnetizing inductance for an IPOP connection of three modules with different magnetizing inductances and same duty cycles.

For an IPOS connection, the average output currents of all modules are equal to $I_{o}$. The output voltage $V_{o}$ is the sum of 
all voltages $V_{o_{k}}$. By substituting (15) in (19) yields

$$
L_{m_{k}}^{2}+\frac{d_{k}}{\sum_{\substack{j=1 \\ j \neq k}}^{N} \frac{d_{j}^{2}}{L_{m_{j}}}} L_{m_{k}}-\frac{R_{o} d_{k}\left(1-d_{k}\right)^{2}}{2 a^{2} f_{s} \sum_{\substack{j=1 \\ j \neq k}}^{N} \frac{d_{j}^{2}}{L_{m_{j}}}}=0 .
$$

Solving (23) for $L_{m_{k}}$ results in:

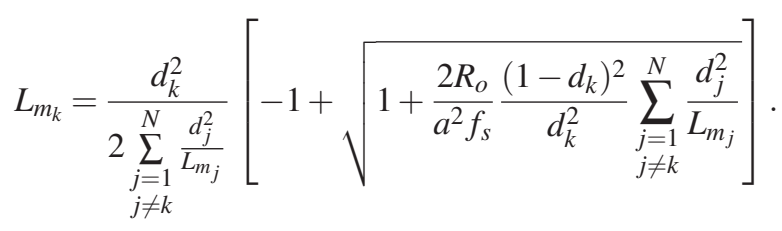

The inductance value that ensures the operation in DCM for output-series connections is represented by (24). Unlike the output parallel connections, in this case there is a maximum value of magnetizing inductance for all possible duty cycle values. The analysis of (24) is shown by Figure 5 for common duty cycles and magnetizing inductances.

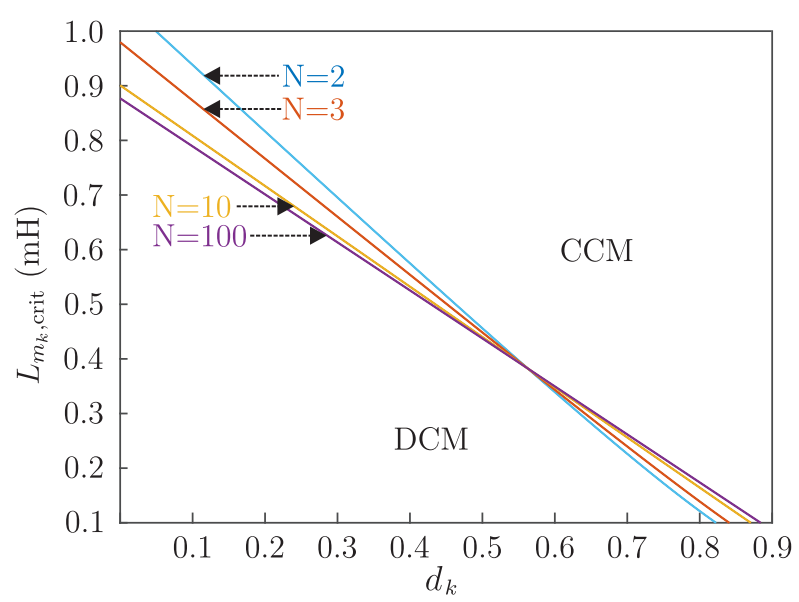

Fig. 5. Critical magnetizing inductance values for a $N$ modules connected in IPOS configuration with same duty cycle $D_{k}$ and magnetizing inductances $L_{m}=382 \mu \mathrm{H}$.

The critical values of inductance decreases slighter than the case of IPOP connections, meaning on a more robust converter in relation to its conduction mode. It should also be noted that the critical values do not significantly vary based on the number of modules $N$.

Equations (21) and (24) for the output parallel and output series connections, respectively, can be used to obtain the critical duty cycle values for each converter. They are useful when each module is controlled by its own duty cycle.

\section{CONTROL-ORIENTED MODELING}

The secondary-side small signal model for a single Flyback module [26] is shown in Figure 6.a. Circuit parameters $r_{D_{j}}$, $j_{D_{j}}$ and $R_{e_{j}}$ are given, respectively, by the following equations:

$$
r_{D_{j}}=\left(\frac{V_{o_{j}}}{V_{i_{j}}}\right)^{2} R_{e_{j}}, j_{D_{j}}=\frac{2 V_{i_{j}}}{d G R_{e_{j}}}, R_{e_{j}}=\frac{2 L_{m_{j}} f_{s}}{d^{2}}
$$

Considering $N$ Flyback modules in an IPOS association, the equivalent secondary-side small signal model can be represented as shown in Figure 6.b. The assumption that all modules have the same constructive parameters leads to the equivalent circuit shown in Figure 7.a. By analyzing the equivalent circuit and considering that $C_{o_{j}}=C_{o \text {, mod }}$, for $j=1,2 \ldots N$, the transfer function that relates a small variation in the output converter voltage $\tilde{v}_{O}$ to a small variation of the common duty cycle $\tilde{d}$ is derived.

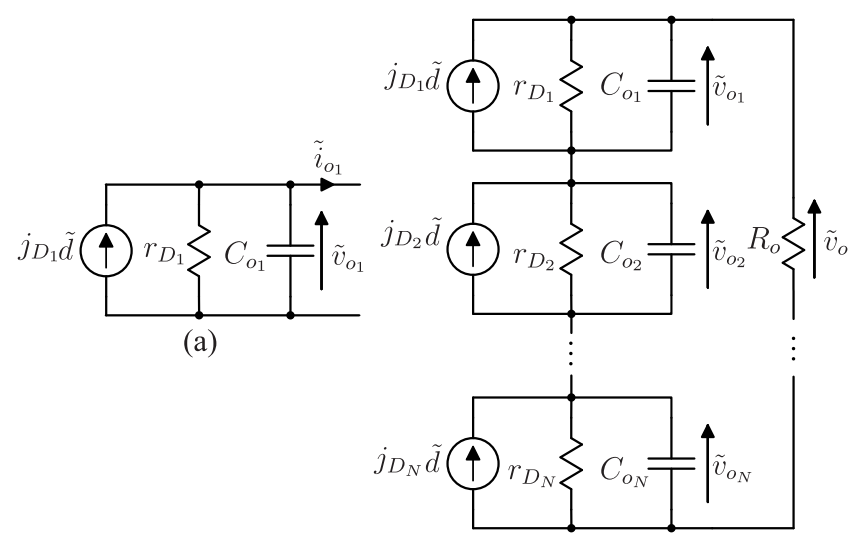

(b)

Fig. 6. Equivalent secondary-side small signal model a) for a single module; b) for an IPOS association.

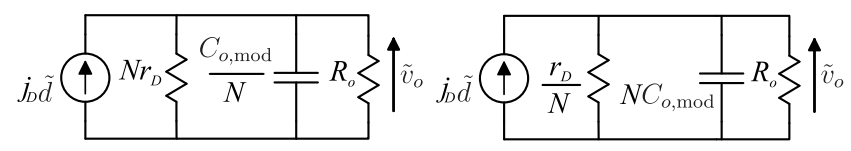

(a)

(b)

Fig. 7. Simplified equivalent secondary-side small signal model a) an IPOS association; b) for an IPOP association.

$$
\frac{\tilde{v}_{o}}{\tilde{d}}=\frac{V_{o} / d}{1+\frac{s C_{o, \bmod } R_{o}}{2 N}} .
$$

If all modules are considered equals, then each of them process the same amount of power. It means that, for an IPOS association, $R_{O}=R_{o, \bmod } N$, with $R_{\mathrm{O}, \bmod }$ equals to the equivalent load resistance of a single module and $V_{o}=V_{o, \bmod } N$, with $V_{o \text {,mod }}$ equals to the averaged output voltage of a single module. Rewriting (26), it is obtained the following transfer function for the modular IPOS Flyback converter:

$$
\frac{\tilde{v}_{o}}{\tilde{d}}=\frac{N V_{o, \bmod } / d}{1+\frac{s C_{o, \bmod } R_{o, \bmod }}{2}} .
$$

An equivalent analysis for an IPOP association provides the circuit shown in Figure 7.b. Assuming similar modules and noting that $R_{o}=R_{o, \bmod } / N$ and $V_{o}=V_{o \text {,mod }}$, it results on the transfer function for the modular IPOP Flyback converter:

$$
\frac{\tilde{v}_{o}}{\tilde{d}}=\frac{V_{o, \bmod } / d}{1+\frac{s C_{o, \bmod } R_{o, \bmod }}{2}} .
$$

Equations (27) and (28) show the similarity between the transfer functions for both modular connections. Indeed, the pole is the same for both, and even more, is equal to the one of a single module. Therefore, assuming that all parameters 
are identical, the dynamic behavior of the converter will not be affected by the association or by the number of modules.

A simplified control strategy for 3 modules in an IPOS connection is sketched in Figure 8. In this paper, the chosen switching frequency is $50 \mathrm{kHz}$, allowing a zerocrossing frequency of $5 \mathrm{kHz}$ and $60^{\circ}$ of phase margin. These requirements can be assured projecting $C_{v_{o}}$ as a ProportionalIntegral (PI) controller.

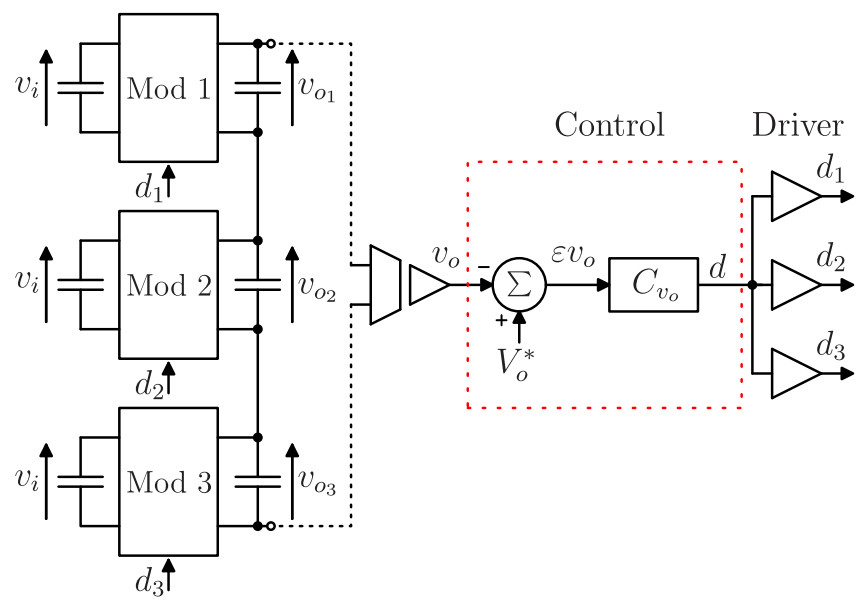

Fig. 8. Simplified control diagram for three modules in an IPOS connection.

\section{SIMULATION AND EXPERIMENTAL RESULTS}

Simulations and experimental tests were performed with a DCM modular Flyback converter composed of three modules in both IPOS and IPOP connections. The parameters of a single module are listed in Table I. The prototype modules are presented in Figure 9. The active switch is a MOSFET 24N60C3 (Infineon) and the output diode is a SiC D06S60C (Infineon).

TABLE I

Module Specifications

\begin{tabular}{lcc}
\hline Parameter & Symbol & Value \\
\hline Input voltage & $V_{i_{j}}$ & $200 \mathrm{~V}$ \\
Output voltage & $V_{o_{j}}$ & $200 \mathrm{~V}$ \\
Rated power & $P_{o_{j}}$ & $200 \mathrm{~W}$ \\
Maximum duty cycle & $d_{\max }$ & 0.45 \\
Magnetizing inductance & $L_{m}$ & $376 \mu \mathrm{H}$ \\
Input capacitor & $C_{i_{j}}$ & $3.03 \mu \mathrm{F}$ \\
Output capacitor & $C_{o_{j}}$ & $2.88 \mu \mathrm{F}$ \\
Switching frequency & $f_{s}$ & $50 \mathrm{kHz}$ \\
\hline
\end{tabular}

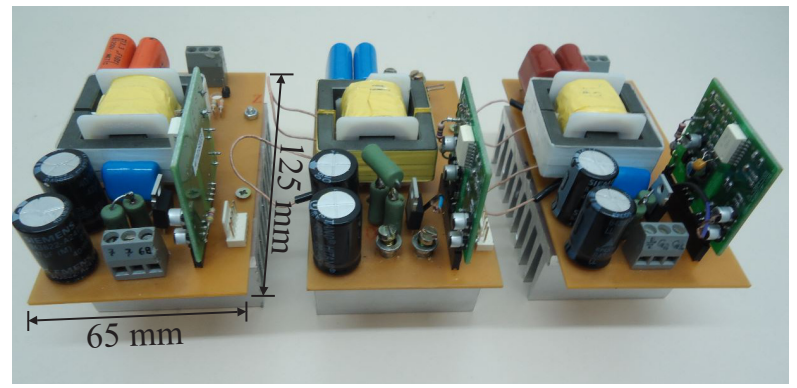

Fig. 9. Prototype composed of 3 Flyback modules.

\section{A. Similar Modules}

The first results demonstrate the performance operating with three approximately equal modules. Table II presents the average values of currents and voltages, obtained via a Wattmeter Yokogawa, model WT 1800, in both IPOS and IPOP connections.

TABLE II

Experimental Results - Average Currents and Voltages

\begin{tabular}{ccccc}
\hline & & Module 1 & Module 2 & Module 3 \\
\hline IPOS & $I_{i_{k}}$ & $983 \mathrm{~mA}$ & $980 \mathrm{~mA}$ & $978 \mathrm{~mA}$ \\
& $V_{o_{k}}$ & $201.0 \mathrm{~V}$ & $200.8 \mathrm{~V}$ & $200.0 \mathrm{~V}$ \\
\hline IPOP & $I_{i_{k}}$ & $983 \mathrm{~mA}$ & $976 \mathrm{~mA}$ & $980 \mathrm{~mA}$ \\
& $I_{o_{k}}$ & $970 \mathrm{~mA}$ & $968 \mathrm{~mA}$ & $968 \mathrm{~mA}$ \\
\hline
\end{tabular}

Figure 10 shows experimental results for three modules in an IPOS connection and Figure 11 for an IPOP connection. Comparing Figure 10.a and Figure 11.a, it is verified a similar behavior on the primary side for the two connections, as expected. Figure 10.b and Figure 11.b demonstrate that, as predicted by equations, the electrical efforts will be balanced. It should be noticed a voltage ringing over the output diode, right after the second operation step. This is a consequence of the interaction between the magnetizing inductance and the parasite capacitance of the switch node, reflected to the secondary side. This operation step characterizes the DCM.

In both connections, the three modules operate with almost identical efficiency. Figure 12 shows the efficiency of one module in an IPOS connection. It has a maximum value of $95.5 \%$ for an output power of $170 \mathrm{~W}$ and $95.3 \%$ for rated power. This result can be extended to the converter.

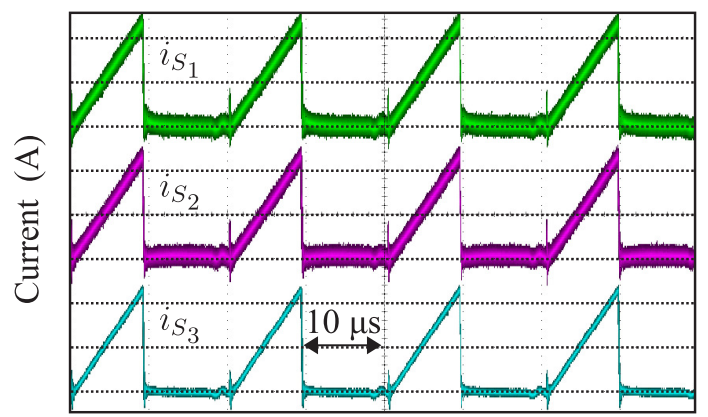

(a)

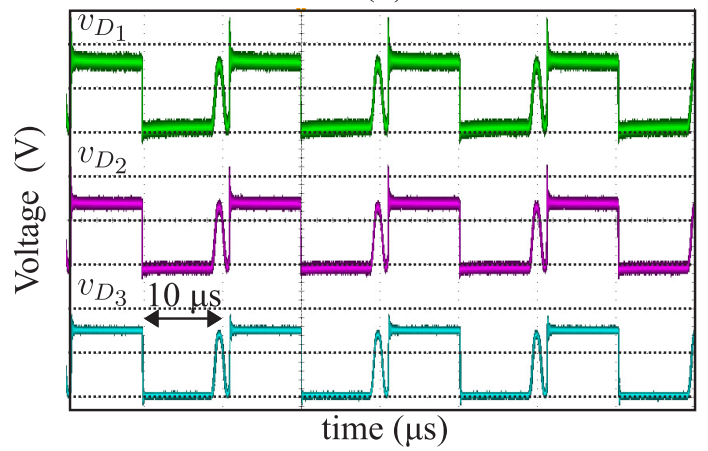

(b)

Fig. 10. Experimental a) primary side current in an IPOS (2 A/div); b) output diode voltage for an IPOS (250 V/div). 


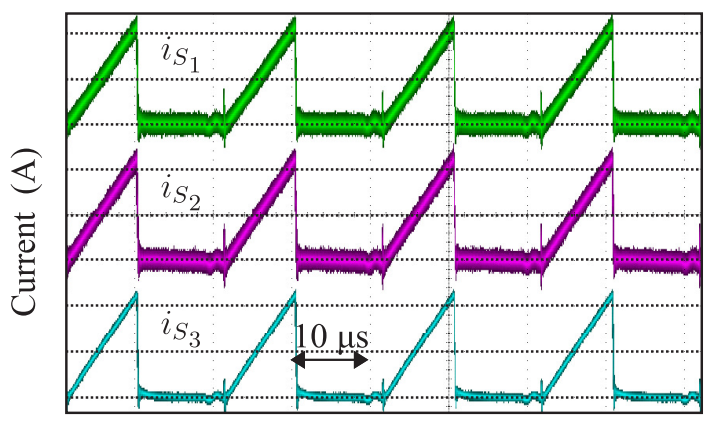

(a)

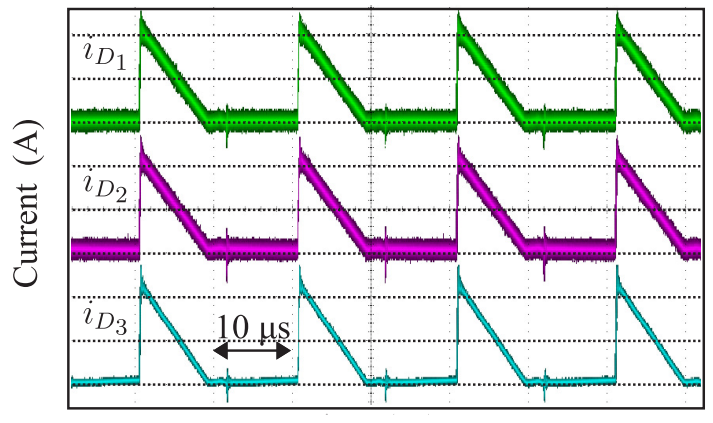

time $(\mu \mathrm{s})$

(b)

Fig. 11. Experimental a) primary side current in an IPOP (2 A/div); b) secundary side current in an IPOP (2 A/div).

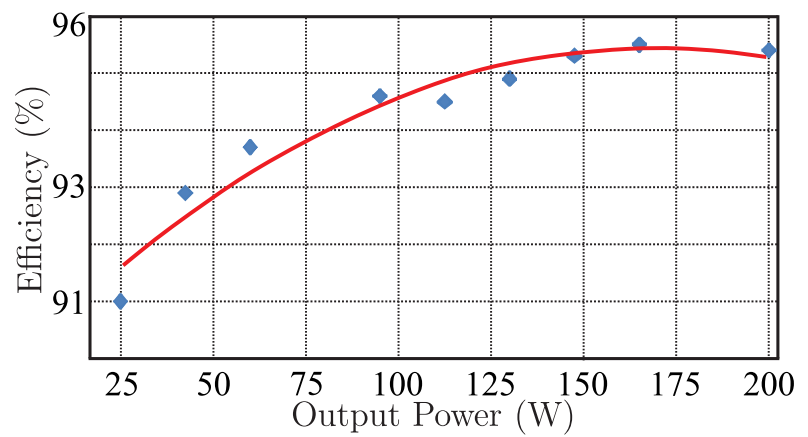

Fig. 12. Efficiency of one module for an IPOP association. The pointed blue line represents the experimental results and the continuous red line the trend curve.

\section{B. Modules with Parameter Mismatches}

Considering non-identical modules, three cases need to be verified: modules with different magnetizing inductances (case 1), duty cycles (case 2) and turns ratio (case 3). Simulation results for average input currents $I_{i_{k}}$ and output voltages $V_{o_{k}}$ are compared with the theoretical imbalance $u_{k}$, as shown in Table III.

TABLE III

Simulation Results for Unbalance in the IPOS Association

\begin{tabular}{|c|c|c|c|c|c|}
\hline & Case & Parameter & $I_{i_{k}} / I_{i}$ & $V_{o_{k}} / V_{o}$ & $u_{k}$ \\
\hline \multirow{9}{*}{$\begin{array}{l}\mathbf{I} \\
\mathbf{P} \\
\mathbf{O} \\
\mathbf{S}\end{array}$} & \multirow{3}{*}{1} & $L_{m_{1}}=357 \mu \mathrm{H}$ & 0.355 & 0.354 & 0.355 \\
\hline & & $L_{m_{2}}=376 \mu \mathrm{H}$ & 0.338 & 0.335 & 0.337 \\
\hline & & $L_{m_{3}}=414 \mu \mathrm{H}$ & 0.307 & 0.308 & 0.307 \\
\hline & \multirow{3}{*}{2} & $d_{1}=0.4275$ & 0.290 & 0.291 & 0.290 \\
\hline & & $d_{2}=0.45$ & 0.322 & 0.323 & 0.321 \\
\hline & & $d_{3}=0.495$ & 0.390 & 0.392 & 0.389 \\
\hline & \multirow{3}{*}{3} & $a_{1}=1$ & 0.333 & 0.333 & 0.333 \\
\hline & & $a_{2}=2$ & 0.333 & 0.333 & 0.333 \\
\hline & & $a_{3}=3$ & 0.333 & 0.333 & 0.333 \\
\hline
\end{tabular}

It is evident in all steady state sharing equations that, while the modules operate in DCM, the input average and output average voltage and current levels, and consequently the imbalance, are not influenced by the turns ratio. Simulation results support this conclusion.

In order to verify the converter imbalances, two experimental tests were carried out on three modules in both IPOS and IPOP connections.

On the first test, the magnetizing inductances in each module are designed as $L_{m_{1}}=392 \mu \mathrm{H}, L_{m_{2}}=450 \mu \mathrm{H}, L_{m_{3}}=$ $382 \mu \mathrm{H}$, with similar duty cycle $D=0.40$. On the second one, $L_{m_{2}}=393 \mu \mathrm{H}$, making the magnetizing inductance similar for all modules. Then, the duty cycles of the modules 2 and 3 are changed to $D_{2}=0.45$ and $D_{3}=0.45$.

In order to ensure the operation in DCM, the critical values for duty cycles and magnetizing inductances were computed using (21) and (24). The values are shown in Table IV and confirm the operation in DCM for all modules.

TABLE IV

Theoretical Critical Values for Experimental Parameters

\begin{tabular}{|c|c|c|c|c|}
\hline \multicolumn{5}{|c|}{ Test 1 - Inductance variations } \\
\hline Module & \multicolumn{2}{|c|}{ IPOP } & \multicolumn{2}{|c|}{ IPOS } \\
\hline & $L_{m, \text { crit }}(\mathrm{mH})$ & $d_{\text {crit }}$ & $L_{m, \text { crit }}(\mu \mathrm{H})$ & $d_{\text {crit }}$ \\
\hline 1 & $\infty$ & 0.490 & 572 & 0.669 \\
\hline 2 & $\infty$ & 0.487 & 557 & 0.614 \\
\hline 3 & $\infty$ & 0.491 & 576 & 0.677 \\
\hline \multicolumn{5}{|c|}{ Test 2 - Duty cycle variations } \\
\hline Module & \multicolumn{2}{|c|}{ IPOP } & \multicolumn{2}{|c|}{ IPOS } \\
\hline & $L_{m, \text { crit }}(\mathrm{mH})$ & $d_{\text {crit }}$ & $L_{m, \text { crit }}(\mu \mathrm{H})$ & $d_{\text {crit }}$ \\
\hline 1 & $\infty$ & 0.518 & 503 & 0.638 \\
\hline 2 & 3.075 & 0.508 & 527 & 0.650 \\
\hline 3 & 2.509 & 0.508 & 531 & 0.661 \\
\hline
\end{tabular}

Table V shows the results for both output series (S) and parallel $(\mathrm{P})$ connections. The variable $u_{t}$ represents the theoretical unbalance values, while $u_{\text {in }}$ and $u_{\text {out }}$ are the experimental unbalances in the input and output, respectively. The relative errors $e_{i n} \%$ and $e_{\text {out }} \%$ between the experimental and theoretical values are calculated for the tests 1 and 2, based on the values presented in Figure 13 and Figure 14, respectively.

TABLE V

Experimental Results

\begin{tabular}{|c|c|c|c|c|c|c|}
\hline \multicolumn{7}{|c|}{ Test 1 - Inductance variations } \\
\hline & $\mathrm{n}$ & $u_{t}$ & $u_{\text {in }}$ & $e_{i n \%}$ & $u_{\text {out }}$ & $e_{\text {out }} \%$ \\
\hline \multirow[t]{3}{*}{$\mathrm{S}$} & 1 & 0.3461 & 0.3547 & 2.4809 & 0.3554 & 2.6938 \\
\hline & 2 & 0.3015 & 0.2932 & -2.7630 & 0.2930 & -2.8151 \\
\hline & 3 & 0.3524 & 0.3521 & -0.0727 & 0.3516 & -0.2372 \\
\hline \multirow[t]{3}{*}{$\mathrm{P}$} & 1 & 0.3461 & 0.3420 & -1.1806 & 0.3431 & -0.8622 \\
\hline & 2 & 0.3015 & 0.3100 & 2.8269 & 0.3091 & 2.5219 \\
\hline & 3 & 0.3524 & 0.3480 & -1.2591 & 0.3478 & -1.3108 \\
\hline \multicolumn{7}{|c|}{ Test 2 - Duty cycle variations } \\
\hline & $\mathrm{n}$ & $u$ & $u_{\text {in }}$ & $e_{i n \%}$ & $u_{\text {out }}$ & $e_{\text {out } \%}$ \\
\hline \multirow[t]{3}{*}{$\mathrm{S}$} & 1 & 0.2816 & 0.2842 & 0.9378 & 0.2845 & 1.0201 \\
\hline & 2 & 0.3555 & 0.3559 & 0.0987 & 0.3557 & 0.0673 \\
\hline & 3 & 0.3629 & 0.3599 & -0.8244 & 0.3598 & -0.8576 \\
\hline \multirow[t]{3}{*}{$\mathrm{P}$} & 1 & 0.2816 & 0.2753 & -2.2393 & 0.2755 & -2.1724 \\
\hline & 2 & 0.3555 & 0.3648 & 2.6267 & 0.3641 & 2.4106 \\
\hline & 3 & 0.3629 & 0.3599 & -0.8356 & 0.3604 & -0.6757 \\
\hline
\end{tabular}




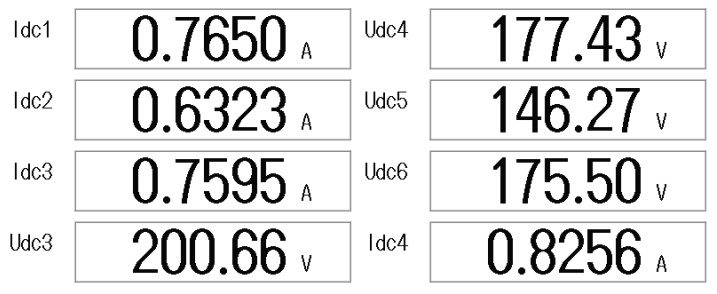

(a)

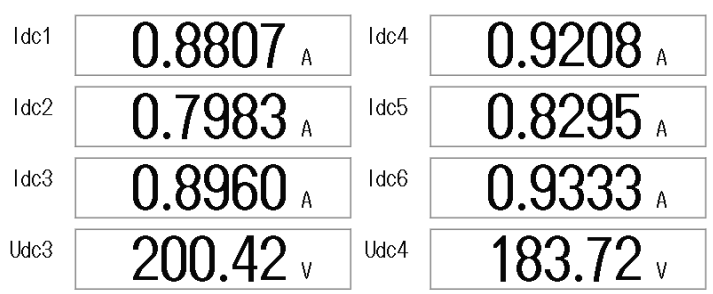

(b)

Fig. 13. Test 1 - Wattimeter measurements for an a) IPOS association ( Full load); b) IPOP association ( Full load). Idc1, Idc2, Idc3 Average input current values on modules 1, 2 and 3, respectively. Udc4/Idc4, Udc5/Idc5, Udc6/Idc6 - Average output voltage/current values on modules 1, 2 and 3, respectively. Udc3 - Average input voltage.

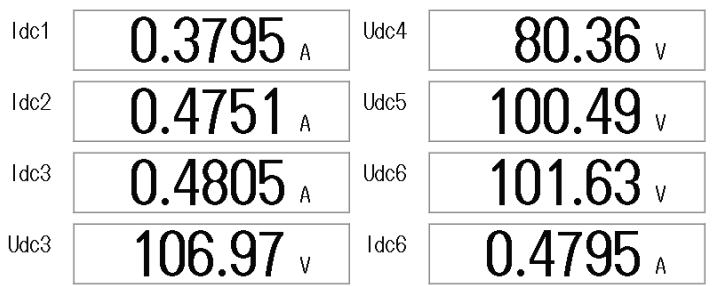

(a)

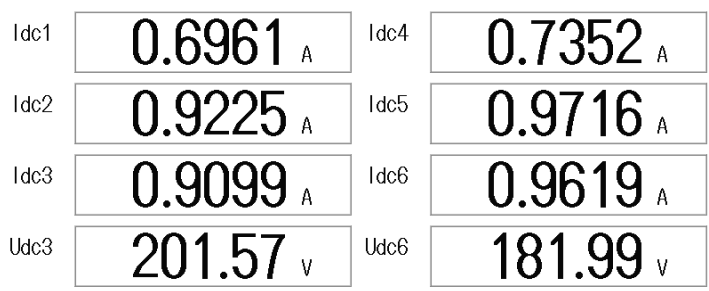

(b)

Fig. 14. Test 2 - Wattimeter measurements for an a) IPOS association ( 25\% load); b) IPOP association ( Full load). Idc1, Idc2, Idc3 Average input current values on modules 1, 2 and 3, respectively. Udc4/Idc4, Udc5/Idc5, Udc6/Idc6 - Average output voltage/current values on modules 1, 2 and 3, respectively. Udc3 - Average input voltage.

\section{Converter Transfer Functions}

The transfer functions are corroborated by simulation results shown in Figure 15. The switched model and the deduced transfer function are compared for a step of roughly $1 \%$ in the duty cycle. Disregarding the high frequency ripple, both show very close dynamic behaviors.

Figure 16.a shows the dynamic response for a step in $d$ for two identical modules in an IPOS connection. They process roughly $150 \mathrm{~W}$ each and have an $8 \mu \mathrm{F}$ output capacitor. An unpredicted low-frequency voltage ripple is observed in $V_{o}$, a consequence of the measurement and zoom via oscilloscope.

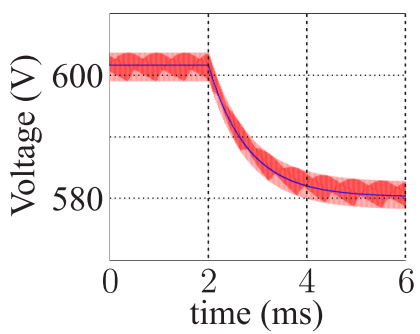

(a)

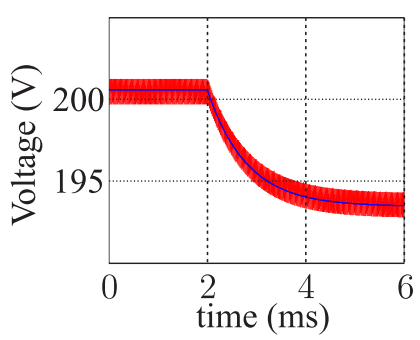

(b)
Fig. 15. Simulation results for the switching model (in red) and deduced transfer function (in blue) for a negative step of $3 \%$ in duty cycle from nominal condition a) in an IPOS association (10 V/div and $2 \mathrm{~ms} / \mathrm{div})$; b) in an IPOP association (5 V/div and $2 \mathrm{~ms} / \mathrm{div})$.

Comparing both simulated and experimental step response a similar dynamics is obtained.

From (27), the time to achieve $95 \%$ of the steady state response should be $5.33 \mathrm{~ms}$. Measuring via oscilloscope, the obtained result is around $5.6 \mathrm{~ms}$.

One of the assumptions for deducing transfer functions was similar parameters for all modules. It must be respected in IPOS connection, however, for IPOP connection an equivalent capacitor can be split among modules without affecting the converter output dynamics.

For the IPOP connection, a third module with a $4.4 \mu F$ capacitor is added. Equation (28) gives a time to achieve $95 \%$ of the steady state response of 4.19 ms. Measuring the waveform shown in Figure 16.b, the obtained result is around $4.3 \mathrm{~ms}$. For both cases, similar settling times and dynamic behavior were found when compared to theoretical and simulated results.

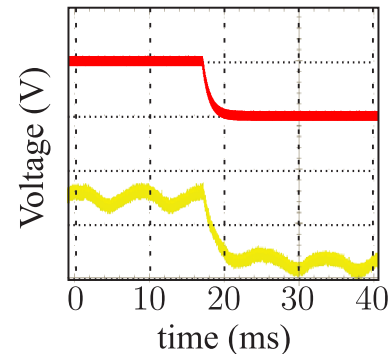

(a)

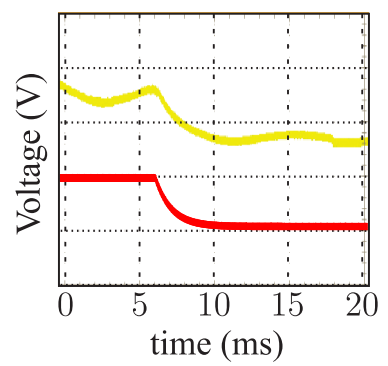

(b)
Fig. 16. Experimental (yellow) and simulated (red) a) IPOS converter output voltage dynamics for a negative step of 0.02 in duty cycle (30 $\mathrm{V} /$ div); b) IPOP converter output voltage dynamics for a negative step of 0.02 in duty cycle. (10 V/div).

By controlling $v_{o}$, it is expected a stable operation after a step in the output load for any number of modules, as seen in the simulated results of Figure 17. Assuming three modules in an IPOS connection and the imbalances given in Table III, after a transient response the module output voltages $v_{o_{1}}, v_{o_{2}}$ and $v_{o_{3}}$ reach a stable point.

\section{CONCLUSION}

Parametric variations among modules may occur for several reasons, whether constructive or due to time or improper handling. This paper quantifies how these mismatches impact on the share of electrical efforts in the input parallel modular connections of Flyback modules operating in DCM. The 


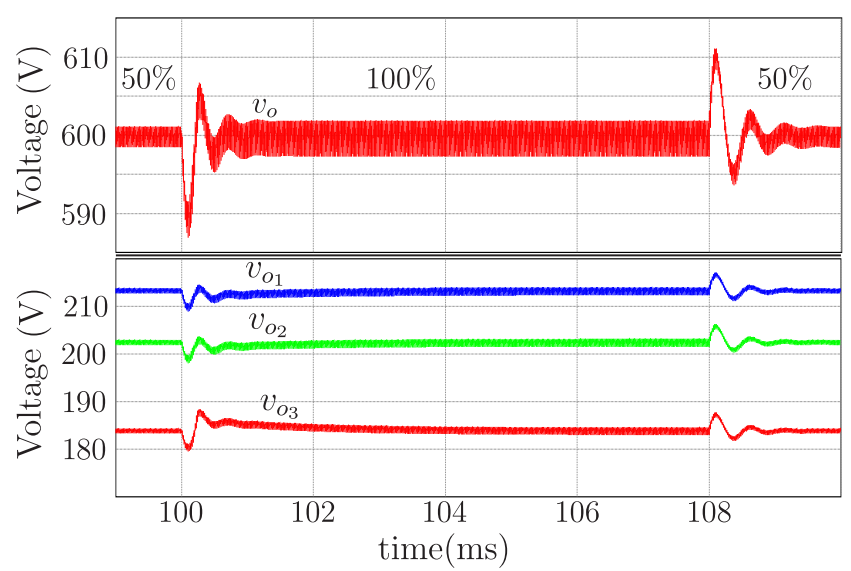

Fig. 17. Simulated response for a step from $50 \%$ to the nominal load, followed by a reduction from the nominal load to $50 \%$. The converter is composed by three modules in an IPOS connection. For the controller, zero-crossing frequency was set in $5 \mathrm{kHz}$ and the phase margin in $60^{\circ}$.

analysis and validation of the steady state imbalance equations were performed. It was shown that current and voltage distributions depend on variations on the duty cycle and on the magnetizing inductance. Then, an analysis of critical values of inductances and duty cycles that ensure the operation in DCM was performed. Once the current or voltage balance is assured, a single control mesh is enough for controlling the output voltage of the converter. Transfer functions for both associations were derived, leading to a simple control strategy whose dynamics do not depend on the association. Finally, the study was corroborated by simulation and by a prototype composed of 3 modules processing $200 \mathrm{~W}$ each. A centralized command was employed, which reduces the duty cycle imbalances among modules and contributes to the load sharing. Also, as expected, magnetizing inductances imbalances leads to imbalances of the same magnitude in the load sharing. The results allow a safer project, once the effect of parametric variations can be taken into account, and contribute to a better understanding of unbalance in modular connections.

\section{ACKNOWLEDGEMENTS}

The authors would like to thank the research funding agencies CAPES and CNPq for the scholarships granted to the students participating in the study.

\section{REFERENCES}

[1] M. A. Pagliosa, R. G. Faust, T. B. Lazzarin, I. Barbi, "Input-series and output-series connected modular single-switch flyback converter operating in the discontinuous conduction mode", IET Power Electronics, vol. 9, no. 9, pp. 1962-1970, 2016.

[2] M. Parchomiuk, R. Strzelecki, K. Zymmer, A. Domino, "Modular power converter topologies for energy storage and electric power distribution systems", in 2017 Progress in Applied Electrical Engineering (PAEE), pp. 1-6, June 2017, doi: 10.1109/PAEE.2017.8009022.
[3] T. B. Lazzarin, I. Barbi, "Design of a 660V / 15 kVA Single-Phase UPS based on Series Connection of LowOutput-Voltage Modular UPS", Eletrônica de Potência - SOBRAEP, vol. 17, pp. 584-591, 2012.

[4] M. Bragard, N. Soltau, S. Thomas, R. De Doncker, "The Balance of Renewable Sources and User Demands in Grids: Power Electronics for Modular Battery Energy Storage Systems", Power Electronics, IEEE Transactions on, vol. 25, no. 12, pp. 3049-3056, Dec 2010.

[5] M. R. Islam, A. M. Mahfuz-Ur-Rahman, M. M. Islam, Y. G. Guo, J. G. Zhu, "Modular Medium-Voltage Grid-Connected Converter With Improved Switching Techniques for Solar Photovoltaic Systems", IEEE Transactions on Industrial Electronics, vol. 64, no. 11, pp. 8887-8896, Nov 2017.

[6] H. Fan, H. Li, "High-Frequency Transformer Isolated Bidirectional DC-DC Converter Modules With High Efficiency Over Wide Load Range for $20 \mathrm{kVA}$ Solid-State Transformer", Power Electronics, IEEE Transactions on, vol. 26, no. 12, pp. 3599-3608, Dec 2011.

[7] G. Kusic, G. Reed, J. Svensson, Z. Wang, "A case for medium voltage DC for distribution circuit applications", in Power Systems Conference and Exposition (PSCE), 2011 IEEE/PES, pp. 1-7, March 2011.

[8] N. Soltau, H. Stagge, R. De Doncker, O. Apeldoorn, "Development and demonstration of a mediumvoltage high-power DC-DC converter for DC distribution systems", in Power Electronics for Distributed Generation Systems (PEDG), 2014 IEEE 5th International Symposium on, pp. 1-8, June 2014.

[9] A. Mohammadpour, L. Parsa, M. Todorovic, R. Lai, R. Datta, L. Garces, "Series-Input Parallel-Output Modular-Phase DC-DC Converter With SoftSwitching and High-Frequency Isolation", Power Electronics, IEEE Transactions on, vol. 31, no. 1, pp. 111-119, Jan 2016.

[10] Y. Matsuoka, K. Takao, K. Wada, M. Nakahara, K. Sung, H. Ohashi, S. Nishizawa, " $2.5 \mathrm{kV}$, $200 \mathrm{~kW}$ bi-directional isolated DC/DC converter for medium-voltage applications", in Power Electronics Conference (IPEC-Hiroshima 2014 - ECCE-ASIA), 2014 International, pp. 744-749, May 2014.

[11] A. Piazzesi, L. Meysenc, "Series connection of 3.3 $\mathrm{kV}$ IGBTs with active voltage balancing", in Power Electronics Specialists Conference, 2004. PESC 04. 2004 IEEE 35th Annual, vol. 2, pp. 893-898 Vol.2, June 2004.

[12] A. Bottion, I. Barbi, "Input-Series and Output-Series Connected Modular Output Capacitor Full-Bridge PWM DC-DC Converter", Industrial Electronics, IEEE Transactions on, vol. 62, no. 10, pp. 6213-6221, Oct 2015.

[13] A. M. Kamath, A. K. G., M. Barai, "Design and implementation of voltage mode digital controller for flyback converter operating in discontinuous conduction mode (DCM)", in 2016 
7th India International Conference on Power Electronics (IICPE), pp. 1-6, Nov 2016, doi: 10.1109/IICPE.2016.8079477.

[14] S. Howimanporn, C. Bunlaksananusorn, "Performance comparison of continuous conduction mode (CCM) and discontinuous conduction mode (DCM) flyback converters", in The Fifth International Conference on Power Electronics and Drive Systems, 2003. PEDS 2003., vol. 2, pp. 1434-1438 Vol.2, Nov 2003.

[15] R. Giri, V. Choudhary, R. Ayyanar, N. Mohan, "Common-duty-ratio control of input-series connected modular DC-DC converters with active input voltage and load-current sharing", Industry Applications, IEEE Transactions on, vol. 42, no. 4, pp. 1101-1111, July 2006.

[16] Q. Lu, Z. Yang, S. Lin, S. Wang, C. Wang, "Research on voltage sharing for input-series-output-series phaseshift full-bridge converters with common-duty-ratio", in IECON 2011 - 37th Annual Conference on IEEE Industrial Electronics Society, pp. 1548-1553, Nov 2011.

[17] L. Qu, D. Zhang, B. Zhang, "Input Voltage Sharing Control Scheme for Input Series and Output Parallel Connected DCâDC Converters Based on Peak Current Control", IEEE Transactions on Industrial Electronics, vol. 66, no. 1, pp. 429-439, Jan 2019.

[18] Z. Zhang, W. Yang, S. Yang, "A modular auxiliary power supply system and its input voltage sharing control strategy", in 2017 IEEE Transportation Electrification Conference and Expo, Asia-Pacific (ITEC Asia-Pacific), pp. 1-6, 2017.

[19] A. J. B. Bottion, I. Barbi, "Input-series and outputseries connected modular full-bridge PWM DC-DC converter with capacitive output filter and common duty cycle", in 2014 11th IEEE/IAS International Conference on Industry Applications, pp. 1-8, Dec 2014.

[20] V. B. Fuerback, M. A. Pagliosa, M. S. Dall'Asta, T. B. Lazzarin, "Modular ISOP Flyback converter: Analysis of auto-balancing mechanism in steady state", in 2017 IEEE 8th International Symposium on Power Electronics for Distributed Generation Systems (PEDG), pp. 1-6, April 2017.

[21] M. A. Pagliosa, T. B. Lazzarin, I. Barbi, "Modular Two-switch Flyback Converter and Analysis of Voltage-balancing Mechanism for Input-series and Output-series Connection", IEEE Transactions on Power Electronics, pp. 1-1, 2018, doi: 10.1109/TPEL.2018.2886072.

[22] J. Shi, L. Zhou, X. He, "Common-Duty-Ratio Control of Input-Parallel Output-Parallel (IPOP) Connected DC-DC Converter Modules With Automatic Sharing of Currents", IEEE Transactions on Power Electronics, vol. 27, no. 7, pp. 3277-3291, July 2012.

[23] L. Wang, T. Zhang, "System performance analysis of the input-parallel and output-series full-bridge converters considering parameter difference", in Electronics Computer Technology (ICECT), 2011 3rd International Conference on, vol. 3, pp. 177-181, April 2011.
[24] Y. Lian, D. Holliday, S. Finney, "Modular inputparallel-output-series DC/DC converter control with fault detection and redundancy", in AC and DC Power Transmission, 11th IET International Conference on, pp. 1-8, Feb 2015.

[25] M. Navidi, A. Hesami Naghshbandi, H. Saberi, S. Hosseini, M. Sabahi, "Parallel input series output DC/DC converter for fuel cell applications", in Power Electronics, Drive Systems and Technologies Conference (PEDSTC), 2014 5th, pp. 181-186, Feb 2014.

[26] R. W. Erickson, D. Maksimovic, Fundamentals of Power Electronics, 2ed ed., Springer, 2001.

\section{BIOGRAPHIES}

Vinícius B. Fuerback, born in 1992 in Cunha Porã, $\mathrm{SC}$, Bachelor of Electrical Engineering from the Federal University of Santa Catarina (UFSC), SC, Florianópolis, Brazil (2017). He is currently studying for his Master's Degree at the Federal University of Santa Catarina.

Matheus S. Dall'Asta, born in 1993 in Curitibanos, SC, Bachelor of Electrical Engineering from the Federal University of Santa Catarina (UFSC), SC, Florianópolis, Brazil (2017). He is currently studying for his Master's Degree at the Federal University of Santa Catarina.

Mauro André Pagliosa, born in 1979 in Bela Vista, MS, is an electrical engineer (2002), master (2005) and doctor in Electrical Engineer (2018) from the Federal University of Santa Catarina (UFSC), Florianópolis, Brazil. He is currently a full professor at the Federal Institute Catarinense, Luzerna, SC.

Telles Brunelli Lazzarin, born in 1979 in Criciúma, SC, is an electrical engineer (2004), master (2006) and doctor in Electrical Engineer (2010) from the Federal University of Santa Catarina (UFSC), Florianópolis, Brazil. He was awarded for one year Post-Doctoral Scholarship (July 2017 to July 2018), working as a researcher guest of the Power Electronics Research Group at Northeastern University. He is currently a full professor at the Federal University of Santa Catarina. Dr. Lazzarin is a member of the SOBRAEP and IEEE. 